\title{
Agricultural machine parts quality control by dynamic non-destructive methods
}

\author{
Alexey N. Beskopylny ${ }^{1, *}$, Viktor B. Rykov ${ }^{2}$, Elena M. Zubrilina ${ }^{1}$, and Andrey D. Chistyakov ${ }^{1}$ \\ ${ }^{1}$ Don State Technical University, pl. Gagarina, 1, Rostov-on-Don, 344010, Russia \\ ${ }^{2}$ State Scientific Establishment "Agriculture research center "Donskoy", North Caucasus Institute of \\ Mechanization and Electrification of Agriculture, 347740 Zernograd, Russia
}

\begin{abstract}
The problem of quality control of agricultural machinery parts by means of dynamic non-destructive methods of impact indentation of conical shape indenters is considered. Quality of the crucial parts is considered from the point of view of a set of mechanical characteristics that determine the strength, hardness, wear resistance and deformability. These characteristics must be known and be able to control on all the details promptly and accurately both in the manufacturing process and in operation. The mechanical characteristics of metals are stochastic in nature. For this purpose, an impact indentation device was developed, and the method based on probabilistic laws of dispersion of mechanical characteristics is obtained.
\end{abstract}

\section{Introduction}

Quality control improvement is very important and widely spread problem concerning large field of manufacturing especially for an imperfect production system subject to both reliability and quality degradations. In [1] the quality control is performed by using $100 \%$ inspection policy to obtain the proportion of defectives. Founded on the quality information feedback, an optimal action is conducted once the proportion reaches a given threshold during production runs on the basis of a stochastic mathematical model.

The determination of the main mechanical properties of agricultural machine parts and elements is one of the most important tasks of machinery improvement at any stage of their operation. When designing it's important to know the most significant mechanical properties, such as yield strength, tensile strength, elongation, and hardness [2,3] which determine the strength, wear resistance and deformability of the most important parts. Recent years the non-destructive testing methods are widely spread in quality management in mechanical engineering, civil engineering [4,5] and metallurgy. There are many researches show the possibility of assessment of the parameters of creep [6] of materials, fatigue [7] and welding residual properties [8].

The model of quality control that is studied in [9] is based on principal-agent theory, which considers the reputational loss due to inferior products and external responsibility identification. After model analysis and simulation verification, the results show that the

\footnotetext{
* Corresponding author: besk-an@ya.ru
} 
optimal quality-control level and market price of agricultural products can be achieved in the agricultural supply chain based on "Internet +" if and only if the information platform's claim to the agricultural producer is less than the agricultural producer's claim to the delivery service provider.

The information modeling of supply chain is constructed in [10] for quality information including environment information, processing procedures and product quality assessments based on principle of quality safety factors.

The thermal analysis system has been developed in [11] to predict the chemical composition in the iron melt. The principle of the technique is the implementation of the characteristics of the cooling curves of sampled iron to correlate with the compositions.

The quality control algorithms of machine parts material must be based on the measurement of a set of mechanical characteristics, such as yield and strength limits, hardness and elongation. The stochastic nature of the properties of steels leads to the need to study the laws of distribution of mechanical characteristics and build a plan of measurements for the correct description of the probabilistic parameters.

\section{Materials and methods}

We consider wide specter of materials of the steel parts of agricultural machines with yield strength $\sigma_{y}$, ultimate strength $\sigma_{u}$, hardness $\mathrm{HV}$ and elongation $\delta$. These mechanical characteristics are random variables

Let us consider the example of the yield point construction of the probability model and the algorithm for estimating the distribution parameters. The random variable $\Theta$, characterizing the strength of the metal (yield strength, tensile strength, etc.). To determine the type of distribution law of a random variable $F(x)=P\{\Theta \leq x\}$, we consider an approach based on the transformation of random variables.

As it is known, the stress-strain curve under uniaxial tension is satisfactorily approximated by the power function. Select a section of this curve on the interval $\sigma \in\left[\sigma_{0} ; \sigma_{B}\right]$

$$
\sigma=\sigma_{0}+A_{0} \varepsilon^{m}
$$

where $\sigma_{0}, A_{0}, m$-parameters of stress-strain curve.

The first derivative

$$
\varepsilon^{\prime}(\sigma)=B_{1}\left(\sigma-\sigma_{0}\right)^{\gamma}
$$

where $B_{1}=\left(m A_{0}^{1 / m}\right)^{-1}, \gamma=m^{-1}-1$.

We introduce a new variable $U=U(\sigma)$ that determines the accumulation of plastic deformation

$$
u=\int_{\sigma_{0}}^{\sigma} \varepsilon^{\prime}(z) d z=B_{0}\left(\sigma-\sigma_{0}\right)^{\gamma+1}
$$

For the new variable $U=U(x)$, the ratio is

$$
P\{\Theta \leq x\}=P\left\{U \leq \int_{\sigma_{0}}^{x} \varepsilon^{\prime}(z) d z\right\} .
$$


If the variable $U$ has a density distribution $f_{l}(u)$, its distribution law

$$
P\{U \leq u\}=F(u)=\int_{0}^{u} f_{1}(x) d x
$$

We consider the function $f_{1}(u) \cong \exp (-u)$, then the distribution law

$$
G(x)=F\left[B_{0}\left(x-\sigma_{0}\right)^{\gamma+1}\right]=\int_{0}^{B_{0}\left(x-\sigma_{0}\right)^{\gamma+1}} \exp (-u) d u=1-\exp \left[-B_{0}\left(x-\sigma_{0}\right)^{\gamma+1}\right]
$$

The obtained distribution is known in the literature as the three-parameter Weibull law, the General form of which

$$
F(x)=1-\exp \left[-\left(\frac{x-\mu}{\beta}\right)^{k}\right]
$$

where $\mu, \beta, k$ are the shift, scale, and shape parameters.

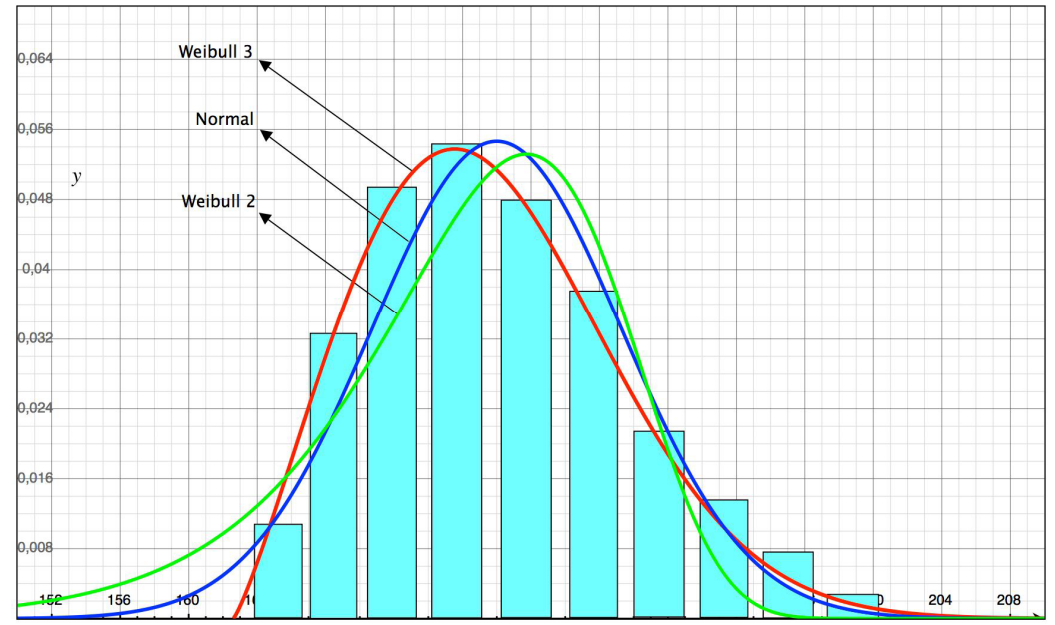

Fig.1. Histogram and probability density distributions for $\sigma_{y}$ when approximation are Weibull with 2 parameters, 3 parameters and Normal distribution.

It is seen (figure 1) that the Weibull law with 3 parameters best describes the distribution of the yield strength of steel.

Consider the relationship between stresses and strains in the form of the proposed E. Voce, which in a simplified form we write

$$
\sigma=\sigma_{0}+A_{0} \exp (\varepsilon)
$$

If from the whole class of exponential distributions, the variable $U$ is described by the normal law with density

$$
f_{1}(u)=\left(2 \pi a^{2}\right)^{-1 / 2} \exp \left[-\frac{u^{2}}{2 a^{2}}\right]
$$


where $a$ is the parameter characterizing the dispersion of the value $U$, the law of distribution of the tensile strength

$$
G(x)=P\{\Theta \leq x\}=\int_{0}^{\ln \left(x-\sigma_{0}\right)-A_{1}} f_{1}(z) d z==\Phi\left[\left(\ln \left(x-\sigma_{0}\right)-A_{1}\right) / a\right]
$$

where $\Phi(\cdot)$ is the Laplace function.

The resulting distribution is log-normal with a shift, a General view of which

$$
F(x)=\frac{1}{a \sqrt{2 \pi}} \int_{\mu}^{x}(\tau-\mu)^{-1} \exp \left\{-\frac{[\ln (\tau-\mu)-b]^{2}}{2 a^{2}}\right\} d \tau
$$

where $\mu, a, \mathrm{~b}$ - parameters.

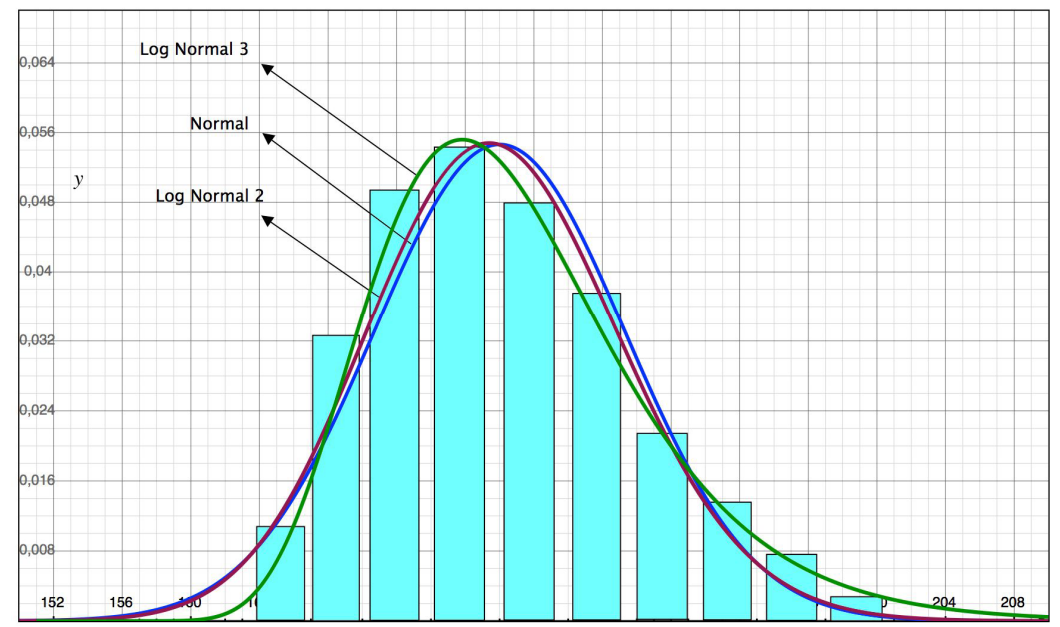

Fig.2. Histogram and probability density distributions for $\sigma_{y}$ when approximation are Lognormal with 2 parameters, 3 parameters and Normal distribution

It is seen (figure 2) that the Lognormal law with 3 parameters best describes the distribution of the yield strength of steel.

The shift parameter in these expressions is the most important characteristic because it shows the minimum strength value. This parameter is necessary to measure and therefore the parameter to judge the quality of manufacturing parts.

\section{Dynamic non-destructive tests}

During manufacture machine elements are treated by complex types thermal, mechanical, chemical or another power, after which the material properties change. The theoretical and experimental studies made it possible to design of an instrument [12] for determining the mechanical properties of steels (Fig. 3), which consist of handle 1, spring 2, striker 3, body 4 , indenter holder 5 , sensor 6 and 9, body part 7, damper 8 .

During measurement striker 3 hits over the indenter holder 5 and sensor 6 registers signal of velocity $V(t)$. The dependences $S(t)$ and $W(t)$ are obtained by integrating and differentiating. 
Numerous experimental studies have shown that with an accuracy of $7 \%$, we can determine the complex mechanical characteristics of materials both in the manufacturing process and during operation.

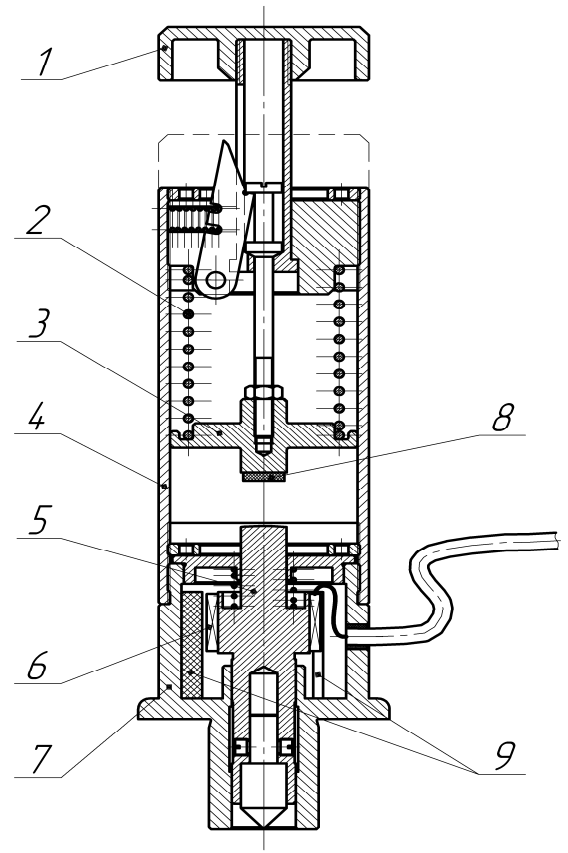

Fig.3. The devise for mechanical properties measurement.

\section{Results and discussion}

For the practical certification of structural steels for mechanical properties, considering the error of their statistical evaluation, the following works should be performed. For each steel grade used by the plant for the manufacture of machine parts, a representative sample of the main strength characteristics: tensile strength, yield strength, elongation and hardness should be extracted from the mechanical test log for a certain pre-accepted period of time. Using a special program based on the algorithms of statistical estimation by the maximum likelihood method, the hypothesis of compliance of these samples with the Weibull laws and the lognormal one with the shift parameter is tested. If the hypothesis of the correspondence of the experimental data of the theoretical curve is correct simultaneously for two laws, the law with the minimum value of the von Mises test should be preferred, or the Weibull law, if the value of the criterion is approximately the same. Such works are carried out approximately once a year, or at modification of technical process of production.

For each steel grade, using a special program based on statistical modeling algorithms, taking into account information about the distribution parameters, for volumes $n=2,5,10$, 15 elements determine the average errors of the minimum mechanical property for all strength characteristics. By the magnitude of these errors, a rational sample size is chosen for quality control and certification. This sample size will correspond to the specific average error of the minimum property evaluation, which is specified in the certificate. As data on certification tests are accumulated, the initial information used as a priori is updated and the control procedure is repeated. 
The results also show that the certification requirements should be different depending on the purpose of the steel. For example, for 17GSU steel operating under impact conditions, it is preferable to tighten the requirements for certification of impact strength and limit the volume to $n=3 \ldots 5$ samples using the formula (4), with an error of $\varepsilon_{\mu}<5 \%$. In these circumstances, for steel 40 the sample size $n=5$ is not enough and must increase.

\section{Conclusions}

The existing methods of quality control and certification of structural steels used in the manufacture of agricultural machines are based on the test of one or two samples from a batch of several hundred tons of finished products, which leads to significant errors in the determination of mechanical properties. To improve the accuracy of the proposed method based on the use of information about the properties of the controlled material on the basis of the theory of extreme sampling terms.

One of the most important indicators of the quality of structural steels is the minimum value of mechanical properties, such as yield and strength limits, elongation, hardness, which is expressed by the shift parameter in the probabilistic laws of Weibull and lognormal. Statistical procedures based on the theory of extreme sampling members are proposed for the effective evaluation of this parameter for small samples.

\section{References}

1. Guo Qing Cheng, Bing Hai Zhou, Ling Li Reliability Engineering \& System Safety, 175, 251-264 (2018)

2. A. Beskopylny, A. Veremeenko, E. Kadomtseva, N. Beskopylnaia, MATEC Web of Conferences 129, 02046 (2017)

3. A Beskopylny, A Veremeenko, B Yazyev, MATEC Web of Conferences 106, 04004 (2017)

4. A Beskopylny, A Lyapin, V Andreev, MATEC Web of Conferences 117, 00018 (2017)

5. A. Beskopylny, A. Lyapin, M. Kadomtsev, A. Veremeenko, Web of Conferences 146, 02013 (2018)

6. S. Litvinov, A. Beskopylny, L. Trush, S. Yazyev, MATEC Web of Conferences 106, 04013 (2017)

7. A Beskopylny, $\mathrm{N}$ Onishkov, $\mathrm{V}$ Korotkin, Advances in Intelligent Systems and Computing 692 184-191 (2017)

8. D. Belen'kii, A. Beskopyl'nyi, N. Vernezi, L. Shamraev, Welding International, 11 , 642-645 (1997)

9. Qiang Shen, Jian Zhang, Yun-xian Hou, Jia-hui Yu, Jin-you Hu, Information Processing in Agriculture, 5, (2018) In press

10. Lu Liu, Xinlei Liu, Guangchen Liu, Information Processing in Agriculture, 5, 47-59 (2018)

11. Sarum Boonmee, Kittirat Worakhut, Materials Today: Proceedings, 5, 3, Part 2, $9497-$ 9505 (2018)

12. D.M. Belenkii, A.N. Beskopylnyi, N.N. Beskopylnyi, E.K. Polibin, B.A. Pesenko Method for determining mechanical characteristics and the device for its implementation. Patent No. 2079831 (1997) 